\title{
Neutral chlorine and molecular hydrogen at high redshift
}

\author{
S. A. Balashev ${ }^{1,2}$, P. Noterdaeme ${ }^{3}$, V. V. Klimenko ${ }^{1,2}$, P. Petitjean $^{3}$, R. Srianand ${ }^{5}$, \\ C. Ledoux ${ }^{4}$ A. V. Ivanchik ${ }^{1,2}$, and D. A. Varshalovich ${ }^{1,2}$ \\ 1 Ioffe Physical-Technical Institute of RAS, Polyteknicheskaya 26, 194021 Saint-Petersburg, Russia \\ e-mail: s.balashev@gmail.com \\ 2 St.-Petersburg Polytechnic University, Polyteknicheskaya 29, 195251 Saint-Petersburg, Russia \\ 3 Institut d'Astrophysique de Paris, CNRS-UPMC, UMR7095, 98bis Bd Arago, 75014 Paris, France \\ ${ }^{4}$ European Southern Observatory, Alonso de Córdova 3107, Vitacura, Casilla 19001, Santiago 19, Chile \\ 5 Inter-University Centre for Astronomy and Astrophysics, Post Bag 4, Ganeshkhind, 411007 Pune, India \\ Received 19 December 2014 / Accepted 28 January 2015
}

\section{ABSTRACT}

\begin{abstract}
Chlorine and molecular hydrogen are known to be tightly linked together in the cold phase of the local interstellar medium through rapid chemical reactions. We present here the first systematic study of this relation at high redshifts using $\mathrm{H}_{2}$-bearing damped Ly $\alpha$ systems (DLAs) detected along quasar lines of sight. Using high-resolution spectroscopic data from VLT/UVES and Keck/HIRES, we report the detection of $\mathrm{Cl}$ I in 9 DLAs (including five new detections) out of 18 high- $z$ DLAs with $N\left(\mathrm{H}_{2}\right) \geq 10^{17.3} \mathrm{~cm}^{-2}$ (including a new $\mathrm{H}_{2}$ detection at $z=3.09145$ towards $\left.\mathrm{J} 2100-0641\right)$ and present upper limits for the remaining 9 systems. We find a $\sim 5 \sigma$ correlation between $N(\mathrm{Cl}$ I $)$ and $N\left(\mathrm{H}_{2}\right)$ with only $\sim 0.2$ dex dispersion over the range $18.1<\log N\left(\mathrm{H}_{2}\right)<20.1$, thus probing column densities 10 times lower than those seen towards nearby stars, roughly following the relation $N(\mathrm{Cl} \mathrm{I}) \approx 1.5 \times 10^{-6} \times N\left(\mathrm{H}_{2}\right)$. This relation between column densities is surprisingly the same at low and high redshift suggesting that the physical and chemical conditions are similar for a given $\mathrm{H}_{2}$ (or $\mathrm{Cl}$ I) column density. In turn, the $N\left(\mathrm{Cl}\right.$ I) $/ N\left(\mathrm{H}_{2}\right)$ ratio is found to be uncorrelated with the overall metallicity in the DLA. Our results confirm that neutral chlorine is an excellent tracer of molecule-rich gas and show that the molecular fraction and/or metallicity in the $\mathrm{H}_{2}$-bearing component of DLA could possibly be much higher than the line-of-sight average values usually measured in DLAs.
\end{abstract}

Key words. cosmology: observations - ISM: clouds - quasars: absorption lines

\section{Introduction}

It has been shown that the global star-formation rate in the Universe gradually increases from $z \sim 10$ to $z \sim 2-3$ and then steeply decreases until the present epoch, $z=0$ (see e.g. Dunlop 2011, and references therein). Because metals are produced by stars, the determination of metal abundance in the gas provides complementary information about star-formation history (Rafelski et al. 2012). This can be done using damped Lyman- $\alpha$ absorption systems (DLAs) that represent the main reservoir of neutral gas at high redshift (Prochaska \& Wolfe 2009; Noterdaeme et al. 2009) and are likely to be located in galaxies or in their close environment (e.g. Krogager et al. 2012). These systems arise mostly in the warm neutral medium (e.g. Petitjean et al. 2000; Kanekar et al. 2014) and have a multicomponent velocity structure, with metal absorption lines spread typically over $100-500 \mathrm{~km} \mathrm{~s}^{-1}$ (Ledoux et al. 1998). In a small fraction of DLAs, the line of sight intercepts cold gas, as traced by molecular hydrogen (e.g. Noterdaeme et al. 2008, 2011; Balashev et al. 2014) and/or $21 \mathrm{~cm}$ absorption (e.g. Srianand et al. 2012). Important progress has been made towards understanding the properties of the gas, for example through deriving physical conditions (Srianand et al. 2005; Noterdaeme et al. 2007a; Jorgenson et al. 2009) and physical extent (Balashev et al. 2011), and the incidence of cold gas in DLAs has been related to other properties such as the metallicity (Petitjean et al. 2006) or the dust content (Ledoux et al. 2003; Noterdaeme et al. 2008). However, owing to the strong saturation of H I Lyman series lines, it remains impossible to directly determine the H I column density associated with the individual cold gas components traced by $\mathrm{H}_{2}$ absorption. Even for metals, whose absorption lines are not saturated, it is very difficult to determine what fraction originates from the cold phase. Difficulties arise as well with the $21 \mathrm{~cm}$ absorption that does not always exactly coincide with $\mathrm{H}_{2}$ absorption (Srianand et al. 2013) although it could be due to the different structures of the optical and radio emitting regions of the background quasars. Out of all the metals, chlorine shows a unique behaviour in the presence of $\mathrm{H}_{2}$. Because the ionization potential of chlorine $(12.97 \mathrm{eV})$ is less than that of atomic hydrogen, chlorine is easily ionized in the diffuse neutral medium. However, this species reacts exothermically with $\mathrm{H}_{2}$ at a very high rate rapidly converting $\mathrm{Cl}^{+}$into $\mathrm{HCl}^{+}$, which subsequently releases neutral chlorine through several channels (Jura 1974; Neufeld \& Wolfire 2009). This process is so efficient that chlorine is completely neutral in the presence of a small amount of $\mathrm{H}_{2}$. In our Galaxy the fact that chlorine abundance anti-correlates with the average number density along the line of sight (Harris \& Bromage 1984; Jenkins et al. 1986) has been interpreted as chlorine depletion. However, models predict and observations indicate that gas with moderate dust content presents negligible depletion of chlorine (e.g. Neufeld \& Wolfire 2009; Savage \& Sembach 1996; Jenkins 2009). Observationally, a tight relation is indeed found between $\mathrm{Cl}$ I and $\mathrm{H}_{2}$ in the local ISM (Jura 1974; Sonnentrucker et al. 2006; Moomey et al. 2012). In this Letter, we present the first study of this relation at high redshift and over a wide range of column densities. 


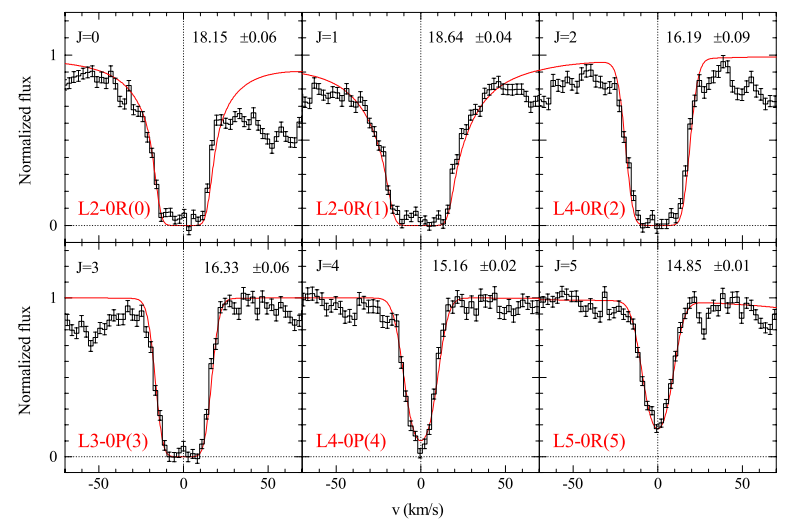

Fig. 1. Voigt profile fits to the newly detected $\mathrm{H}_{2}$ absorption lines from rotational levels $J=0$ to $J=5$ at $z=3.091485$ towards J 2100-0641. The column densities are indicated (in $\log \left(\mathrm{cm}^{-2}\right)$ ) in the top right corner of each panel.

\section{Data sample and measurements}

Since the first detection by Levshakov \& Varshalovich (1985), about two dozen $\mathrm{H}_{2}$ absorption systems have been detected at high redshifts in quasar spectra. The detection limit of the strongest $\mathrm{Cl}$ I absorption line (1347 $\AA, f=0.0153$, Schectman et al. 1993) in a high quality spectrum $(S / N \sim 50, R \sim 50000)$ corresponds to $N(\mathrm{ClI}) \sim 10^{12} \mathrm{~cm}^{-2}$. The solar abundance of chlorine is $10^{-6.5}$ that of hydrogen (Asplund et al. 2009) and given previous measurements of $N(\mathrm{Cl} \mathrm{I}) / 2 N\left(\mathrm{H}_{2}\right)$ (e.g. Moomey et al. 2012) we conservatively limit our study to systems with $N\left(\mathrm{H}_{2}\right) \gtrsim 10^{17} \mathrm{~cm}^{-2}$.

Redshifts, $\mathrm{HI}$ and $\mathrm{H}_{2}$ column densities, and metallicities were mainly taken from the literature and are based on VLT/UVES, Keck/HIRES, or HST/STIS data. We refitted $\mathrm{H}_{2}$ absorption systems towards Q 2123-0050 and Q J2340-0053 to take into account the positions of the detected $\mathrm{Cl}$ I components. We also detect a new $\mathrm{H}_{2}$ absorption system in the $z=3.09$ DLA towards J 2100-0641 in which Jorgenson et al. (2010) have reported the presence of neutral carbon. Indeed, C I is known to be an excellent indicator of the presence of molecules (e.g. Srianand et al. 2005). We used the MAKEE package (Burles) to reduce archival data from this quasar obtained in 2005, 2006, and 2007 under programs U17H (PI: Prochaska), G400H (PI: Ellison), and U149Hr (PI: Wolfe). We have found strong $\mathrm{H}_{2}$ absorption lines from rotational levels up to $J=5$ (see Fig. 1) at $z=3.091485$ with a total column density of $\log N\left(\mathrm{H}_{2}\right)=$ $18.76 \pm 0.03$.

For all systems we retrieved data from the VLT/UVES or the Keck/HIRES archives. We reduced the data and fitted the lines using profile fitting. Neutral chlorine is detected in nine DLAs (Fig. 2). Four detections were already reported in the literature: Q 1232+082 (Balashev et al. 2011), Q 0812-3208 (Prochaska et al. 2003), Q 1237+0647 (Noterdaeme et al. 2010), and Q 2140-0321 (Noterdaeme et al. 2015). The remaining five are new detections. We measured upper-limits of $N(\mathrm{Cl}$ I $)$ for the remaining nine systems. We used mainly the $1347 \AA \mathrm{Cl}$ I line. Whenever possible, we also used $\mathrm{Cl}$ I lines at $1088 \AA$, $1188 \AA$, $1084 \AA, 1094 \AA$, and $1085 \AA$, with oscillator strength from Schectman et al. (1993), Morton (2003), Sonnentrucker et al. (2006), and Oliveira \& Hébrard (2006), respectively.

Table 1 summarizes the results of $\mathrm{Cl}$ I measurements. We have kept all components with $\log N\left(\mathrm{H}_{2}\right)>17$. We did not use two known $\mathrm{H}_{2}$ absorption systems towards Q 0013-0029 and

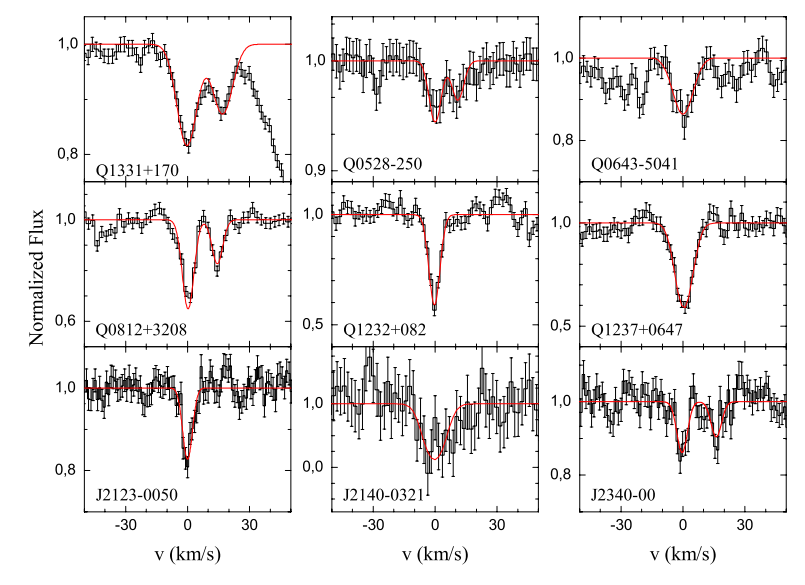

Fig. 2. Voigt profile fits to $\mathrm{Cl}$ I $\lambda 1347$ absorption lines associated with high- $z$ strong $\mathrm{H}_{2}$ absorption systems.

J 091826.16+163609.0 since $\mathrm{H}_{2}$ column densities in these systems are not well defined.

\section{Results}

Figure 3 shows the $\mathrm{Cl}$ I column density, $N(\mathrm{Cl}$ I $)$, versus $N\left(\mathrm{H}_{2}\right)$ and compares our high- $z$ measurements to those obtained in the local ISM using the Copernicus satellite (Moomey et al. 2012). As can be seen, our high- $z$ measurements extend the relation to $\mathrm{H}_{2}$ column densities ten times smaller than those measured in the local ISM. A very close correlation $(r=0.95)$ between Cl I and $\mathrm{H}_{2}$ has been found over the entire $N\left(\mathrm{H}_{2}\right)$ range. It is striking that measurements at high and low redshifts are indistinguishable in the overlapping regime $\left(\log N\left(\mathrm{H}_{2}\right) \sim 19-20.2\right)$. The correlation is seen over about three orders of magnitude in column density with a dispersion of only about 0.2 dex. A least-squares bisector linear fit provides a slope of 0.83 and 0.87 for the high $-z$ and $z=0$ data, respectively, with an almost equal normalization $\left(\log N(\mathrm{Cl} \mathrm{I}) \approx 13.7\right.$ at $\left.\log N\left(\mathrm{H}_{2}\right)=20\right)$. We note that the upper limits on $N(\mathrm{Cl}$ I $)$ lie mostly at the low $N\left(\mathrm{H}_{2}\right)$ end and are least constraining since they are compatible with the values expected from the above relation. For this reason, we will not consider them further in the discussion but still include them in the figures for completeness. The slopes are less than one, meaning that the $\mathrm{Cl} \mathrm{I} / \mathrm{H}_{2}$ ratio slightly decreases with increasing $N\left(\mathrm{H}_{2}\right)$. This is unlikely to be due to conversion of $\mathrm{Cl}$ into $\mathrm{H}_{2} \mathrm{Cl}^{+}$and/or $\mathrm{HCl}$, since chlorine chemistry models (Neufeld \& Wolfire 2009) and measurements (e.g. towards Sgr B2(S), Lis et al. 2010) show that in diffuse molecular clouds only $\sim 1 \%$ of chlorine is in the molecular form. $A<1$ slope could in principle be due to dust depletion. However, there is no trend for increasing $\mathrm{Cl}$ depletion with increasing $\mathrm{H}_{2}$ or $\mathrm{Cl}$ I column densities in Galactic clouds (see Moomey et al. 2012). In addition, for high redshift measurements, elemental abundance patterns (Noterdaeme et al. 2008) as well as direct measurements (e.g. Noterdaeme et al. 2010) indicate $A_{v}<0.2$ when modelling of chlorine chemistry (Neufeld $\&$ Wolfire 2009) shows that for such low extinction $\left(A_{v}<1\right)$ all chlorine is in the gas phase.

A possibility is thus that the molecular fraction in the gas probed by ClI is slightly increasing with increasing $N\left(\mathrm{H}_{2}\right)$. It can be expected since $\mathrm{H}_{2}$ self-shielding increases while $\mathrm{Cl}$ I is already completely in the neutral form. Finally, the similarity between our Galaxy and high- $z$ measurements at $\log N\left(\mathrm{H}_{2}\right) \geq 19$ might indicate that the chemical and physical conditions in the 
Table 1. Measurements of $\mathrm{Cl}$ I in strong $\mathrm{H}_{2}$ absorption systems at high redshift.

\begin{tabular}{|c|c|c|c|c|c|c|c|c|c|c|c|}
\hline Quasar & $z_{\mathrm{em}}$ & $z_{\text {DLA }}$ & $\log N(\mathrm{H} \mathrm{I})$ & {$[\mathrm{X} / \mathrm{H}]$} & $\mathrm{X}$ & Ref. & $z_{\mathrm{H} 2}$ & $\log N\left(\mathrm{H}_{2}\right)$ & $\log N(\mathrm{Cl} \mathrm{I})$ & $b\left(\mathrm{~km} \mathrm{~s}^{-1}\right)$ & {$\left[\mathrm{Cl} \mathrm{I} / \mathrm{H}_{2}\right]$} \\
\hline Q 0027-1836 & 2.56 & 2.40 & $21.75 \pm 0.10$ & $-1.63 \pm 0.10$ & $\mathrm{Zn}$ & 1 & 2.40183 & $17.30 \pm 0.07$ & $<12.71$ & - & $<1.61$ \\
\hline Q $0405-4418$ & 3.02 & 2.59 & $21.75 \pm 0.10$ & $-1.12 \pm 0.10$ & $\mathrm{Zn}$ & 2 & 2.59475 & $18.14 \pm 0.07$ & $<12.71$ & _- & $<0.77$ \\
\hline \multirow[t]{2}{*}{ Q $0528-2505$} & 2.77 & 2.81 & $21.35 \pm 0.07$ & $-0.91 \pm 0.07$ & $\mathrm{Zn}$ & 3 & 2.81098 & $18.11 \pm 0.02$ & $11.92 \pm 0.08$ & $4.1 \pm 1.5$ & $0.01 \pm 0.08$ \\
\hline & & & & & & & 2.81112 & $17.85 \pm 0.02$ & $11.73 \pm 0.11$ & $4.2 \pm 2.0$ & $0.08 \pm 0.11$ \\
\hline Q $0551-3637$ & 2.32 & 1.96 & $20.70 \pm 0.08$ & $-0.35 \pm 0.08$ & $\mathrm{Zn}$ & 4 & 1.96214 & $17.42^{+0.45}$ & $<12.40$ & - & $<1.54$ \\
\hline Q J0643-5041 & 3.09 & 2.66 & $21.03 \pm 0.08$ & $-0.91 \pm 0.09$ & $\mathrm{Zn}$ & 5 & 2.65860 & $18.54 \pm 0.01$ & $12.51 \pm 0.05$ & $5.8 \pm 1.4$ & $0.17 \pm 0.05$ \\
\hline \multirow[t]{2}{*}{ Q J0812+3208 } & 2.7 & 2.63 & $21.35 \pm 0.10$ & $-0.81 \pm 0.10$ & $\mathrm{Zn}$ & 6 & 2.62628 & $18.84 \pm 0.06$ & $12.79 \pm 0.05$ & $2.0 \pm 0.6$ & $0.15 \pm 0.08$ \\
\hline & & & & & & & 2.62644 & $19.93 \pm 0.01$ & $13.78 \pm 0.27$ & $0.17 \pm 0.05$ & $0.05 \pm 0.27$ \\
\hline \multirow[t]{2}{*}{ Q J0816+1446 } & 3.84 & 3.29 & $22.00 \pm 0.10$ & $-1.10 \pm 0.10$ & $\mathrm{Zn}$ & 7 & 3.2874 & $18.62 \pm 0.18$ & $<13.65$ & - & $<1.23$ \\
\hline & & & & & & & 3.2 & 17. & $<1$ & - & \\
\hline Q $1232+0815$ & 2.57 & 2.34 & $20.90 \pm$ & $-1.35 \pm 0.12$ & $\mathrm{~S}$ & 8 & 72 & 0.10 & 13.49 & $0.8 \pm$ & $0.12 \pm 0.13$ \\
\hline Q J1237+0647 & 2.78 & 2.69 & $20.00 \pm$ & $+0.34 \pm 0.12$ & $\mathrm{Zn}$ & 9 & 2.6 & .13 & 13.0 & $4.5 \pm 0.4$ & 0.13 \\
\hline Q $1331+170$ & 2.08 & 1.78 & 21.1 & $-1.22 \pm 0.10$ & $\mathrm{Zn}$ & 10,11 & 1.77636 & \pm 0.10 & 12.87 & $5.7 \pm 0.5$ & $-0.64 \pm 0.10$ \\
\hline Q J1439+1118 & 2.58 & 2.42 & $20.10 \pm 0.10$ & $+0.16 \pm 0.11$ & $\mathrm{Zn}$ & 12 & 84 & .04 & 25 & - & 0.07 \\
\hline \multirow[t]{2}{*}{ Q $1441+2737$} & 4.42 & 4.22 & $20.95 \pm 0.08$ & $-0.63 \pm 0.10$ & $\mathrm{~S}$ & 13 & 4.22401 & 18.05 & 86 & - & $<1.01$ \\
\hline & & & & & & & 4.2 & 17 & $<1$ & - & $<0$ \\
\hline Q $1444+0126$ & 2.21 & 2.09 & $20.25=$ & $-0.80 \pm 0.09$ & $\mathrm{Zn}$ & 14 & 2.08 & .11 & $<1$ & - & $<0.46$ \\
\hline Q J2100-0641 & 3.14 & 3.09 & $21.05 \pm 0.15$ & $-0.73 \pm 0.15$ & $\mathrm{Si}$ & 15 & 3.09149 & 18.76 & $<12.86$ & - & $<0.3$ \\
\hline Q J2123-0050 & 2.26 & 2.06 & $19.18 \pm 0.15$ & $-0.19 \pm 0.10$ & S & 16 & 2.05933 & $18.09 \pm 0.02$ & $12.27 \pm 0.06$ & $2.6 \pm 0.5$ & $0.38 \pm 0.06$ \\
\hline Q J2140-0321 & 2.48 & 2.34 & $22.40 \pm 0.10$ & $-1.05 \pm 0.13$ & $\mathrm{P}$ & 17 & 2.33995 & $20.13 \pm 0.07$ & $13.67 \pm 0.15$ & $5-10$ & $-0.26 \pm 0.18$ \\
\hline \multirow[t]{2}{*}{ Q J2340-0053 } & 2.09 & 2.05 & $20.35 \pm 0.15$ & $-0.92 \pm 0.03$ & $\mathrm{Zn}$ & 15 & 2.05456 & $18.07 \pm 0.06$ & $12.25 \pm 0.15$ & $0.8 \pm 0.6$ & $0.38 \pm 0.16$ \\
\hline & & & & & & & & 18.25 & 12.13 & $1.0 \pm 1.0$ & $0.08 \pm 0.19$ \\
\hline \multirow[t]{2}{*}{ Q 2348-0108 } & 3.01 & 2.43 & $20.50 \pm 0.10$ & $-0.62 \pm 0.10$ & $\mathrm{~S}$ & 18,19 & 2.42688 & $18.12 \pm 0.37$ & $<13.86$ & - & $<1.94$ \\
\hline & & & & & & & 2.42449 & 17.5 & $<1$ & - & $<0.81$ \\
\hline
\end{tabular}

References. (1) Noterdaeme et al. (2007a); (2) Ledoux et al. (2003); (3) Noterdaeme et al. (2008); (4) Ledoux et al. (2002); (5) Albornoz Vásquez et al. (2014); (6) Jorgenson et al. (2009); (7) Guimarães et al. (2012); (8) Balashev et al. (2011); (9) Noterdaeme et al. (2010); (10) Carswell et al. (2011); (11) Balashev et al. (2010); (12) Srianand et al. (2008); (13) Ledoux et al. (2006); (14) Ledoux et al. (2003); (15) Jorgenson et al. (2010); (16) Malec et al. (2010); (17) Noterdaeme et al. (2015); (18) Petitjean et al. (2006); (19) Noterdaeme et al. (2007b).

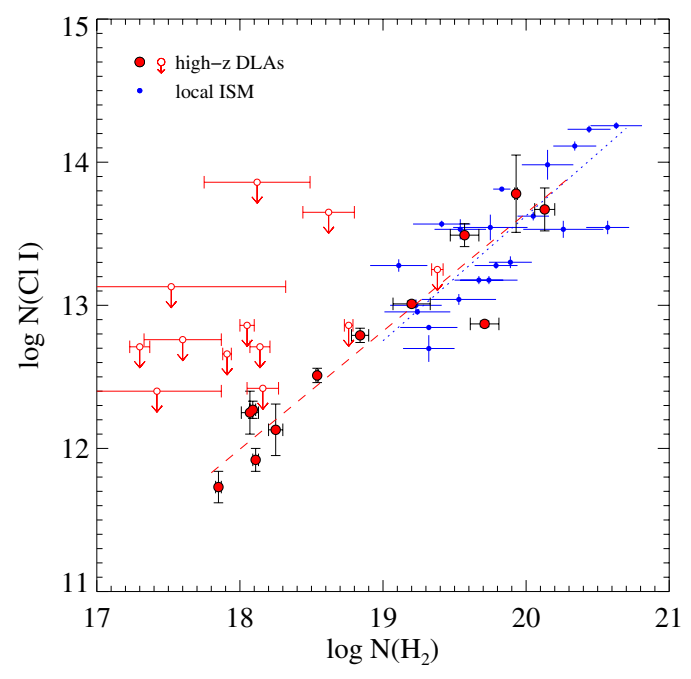

Fig. 3. Column densities of $\mathrm{Cl}$ I versus that of $\mathrm{H}_{2}$. The red and blue points indicate the measurements at high redshift (this work) and in our Galaxy (Moomey et al. 2012), respectively. The straight dashed and dotted lines show the respective least-squares bisector fits to the data.

cold gas can be similar, otherwise fine tuning would be required between the different factors that affect the $N(\mathrm{Cl} \mathrm{I})$-to- $N\left(\mathrm{H}_{2}\right)$ ratio (e.g. number density, metallicity, dust content, and UV flux).

Before continuing further, we note that in $\mathrm{H}_{2}$-bearing gas, chlorine is found exclusively in the neutral form (i.e. $N(\mathrm{Cl})=$ $N(\mathrm{Cl}$ I) ) (e.g. Jura 1974). Since we expect that all chlorine is in gas-phase $^{1}$, the abundance of chlorine, $[\mathrm{Cl} / \mathrm{H}]$, in $\mathrm{H}_{2}$-bearing gas can be expressed as

$[\mathrm{Cl} / \mathrm{H}]=\left[\mathrm{Cl} \mathrm{I} / \mathrm{H}_{2}\right]+\log f$,

where

$\left[\mathrm{Cl} \mathrm{I} / \mathrm{H}_{2}\right]=\log \left(\frac{N(\mathrm{Cl} \mathrm{I})}{2 N\left(\mathrm{H}_{2}\right)}\right)-\log \left(\frac{\mathrm{Cl}}{\mathrm{H}}\right)_{\odot}$

and $f=2 N\left(\mathrm{H}_{2}\right) /\left(2 N\left(\mathrm{H}_{2}\right)+N(\mathrm{HI})\right)$ is the molecular fraction. Therefore, the ratio $\left[\mathrm{Cl} / \mathrm{H}_{2}\right]$ gives a direct constraint on the chlorine-based metallicity of $\mathrm{H}_{2}$-bearing gas provided the molecular fraction of $\mathrm{H}_{2}$-bearing gas is known. Conversely, if a constraint can be put on the actual chlorine abundance, $\left[\mathrm{Cl} \mathrm{I} / \mathrm{H}_{2}\right]$ can provide an estimate of the amount of $\mathrm{HI}$ present in $\mathrm{H}_{2}$-bearing gas. If $\mathrm{Cl}$ is depleted onto dust grains then the mentioned estimates of metallicity and molecular fraction will have to be corrected from the $\mathrm{Cl}$ depletion factor.

Figure 4 shows $\left[\mathrm{Cl} \mathrm{I} / \mathrm{H}_{2}\right]$ as a function of the overall metallicity for DLAs (given in Table 1) at high redshift or as a function of $[\mathrm{Cl} / \mathrm{H}]$ for clouds in our Galaxy. Since $f \leq 1,\left[\mathrm{Cl} \mathrm{I} / \mathrm{H}_{2}\right]$ gives an upper limit on the metallicity in $\mathrm{H}_{2}$-bearing gas, which is found to be roughly equal to or less than solar metallicity. For 13 out of $21 \mathrm{Cl}$ I bearing clouds in our Galaxy, associated Cl II was measured (Moomey et al. 2012). Therefore, we have estimated the overall chlorine abundance $[\mathrm{Cl} / \mathrm{H}]$ of these clouds as $(N(\mathrm{Cl} \mathrm{I})+N(\mathrm{Cl}$ II $)) /\left(N(\mathrm{H} \mathrm{I})+2 N\left(\mathrm{H}_{2}\right)\right)$ (see Fig. 4). Unfortunately, for high redshift DLAs, not only is $\mathrm{Cl}$ II not detected, but DLAs also contain several $\mathrm{HI}$ clouds so that chlorine abundance of

1 The presence of $\mathrm{Cl}$ II in the outer envelope of the $\mathrm{H}_{2}$ cloud is not excluded, but it does not influence our derivation. 


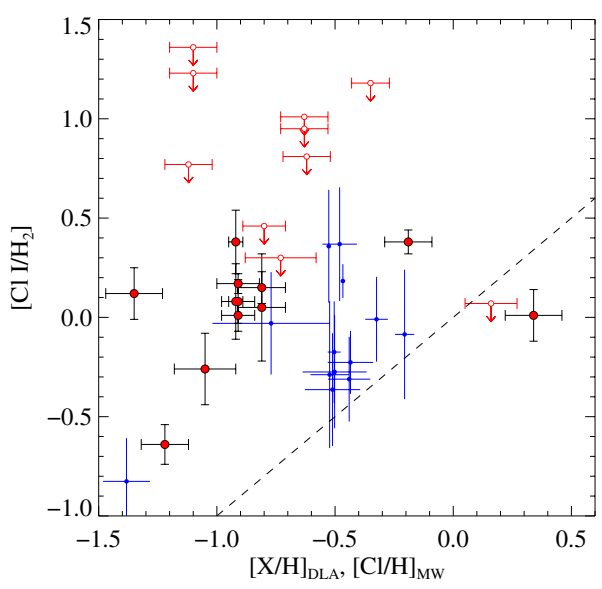

Fig. 4. $\left[\mathrm{Cl} \mathrm{I} / \mathrm{H}_{2}\right]$ as a function of the overall metallicity for high- $z$ DLAs (red points) and the chlorine-based metallicity for Milky Way clouds (blue points). The dashed line represents the one-to-one relation.

the very cloud of interest cannot be measured. Therefore, we consider the overall metallicity (averaged over velocity components) measured using another non-depleted element (usually $\mathrm{Zn}$ or $\mathrm{S}$, see Table 1). In Fig. 4 it can be seen that the $\left[\mathrm{Cl} / / \mathrm{H}_{2}\right]$ ratio is likely not correlated with the overall metallicity of the DLA (Pearson correlation coefficient 0.3 at 0.3 significance level). For Milky Way clouds it can be seen that $[\mathrm{Cl} / \mathrm{H}]$ is typically one third solar, which can be interpreted as evidence for chlorine depletion (Moomey et al. 2012).

The large difference between $\left[\mathrm{Cl} / \mathrm{H}_{2}\right]$ and $[\mathrm{X} / \mathrm{H}]_{\text {DLA }}$ for the high redshift clouds can be explained by a molecular fraction $f<1$ in $\mathrm{H}_{2}$-bearing clouds, by a higher metallicity in the $\mathrm{H}_{2}$ bearing gas compared to the overall DLA metallicity, or by both effects. If we assume that the metallicity in the $\mathrm{H}_{2}$-bearing gas is equal to the overall DLA metallicity we find (using Eq. (1)) that the molecular fraction in the $\mathrm{H}_{2}$-bearing gas is typically an order of magnitude higher than the overall inferred DLA molecular fraction. Interestingly, two systems sitting close to the oneto-one relation are those where $\mathrm{CO}$ molecules have been detected (Q 1439+1118 and Q 1237+0647). In such systems, the $\mathrm{H}_{2}$ component is probably fully molecularized and its metallicity is close to the overall metallicity of the DLA.

\section{Conclusion}

We have studied the neutral chlorine abundance in high redshift $(z \sim 2-4)$ strong $\mathrm{H}_{2}$-bearing DLAs with $\log N\left(\mathrm{H}_{2}\right)>17.3$. These systems arise in the cold neutral medium of galaxies in the early Universe. We have used 17 systems from the literature and also present a new $\mathrm{H}_{2}$ detection at $z=3.09145$ in the spectrum of J 2100-0641. We have detected $\mathrm{Cl}$ I absorption lines in half of these systems, including five new detections. The derived upper limits for $\mathrm{N}(\mathrm{Cl} \mathrm{I})$ for the remaining systems are shown to be consistent with the behaviour of the overall population. Our measurements extend the $\mathrm{Cl}-\mathrm{I}_{2}$ relation to lower column densities than measurements towards nearby stars. We show that there is a $5 \sigma$ correlation between the column densities of both species over the range $18.1<\log N\left(\mathrm{H}_{2}\right)<20.1$ with indistinguishable behaviour between high and zero redshift systems. This suggests that at a given $N\left(\mathrm{H}_{2}\right)$ the physical conditions are likely similar in our Galaxy and high- $z$ gas, in spite of possible differences in the dust depletion levels. As we expect the $\mathrm{Cl}$ to be depleted less in the high- $z$ absorbers studied here, we use the abundance of chlorine with respect to $\mathrm{H}_{2}$ to constrain the molecular fraction and the metallicity in $\mathrm{H}_{2}$-bearing gas. Our results suggest that the molecular fraction and/or the metallicity in the $\mathrm{H}_{2}$ - and $\mathrm{Cl}$ I-bearing components could be much higher than the mean value measured over the whole DLA system. This implies that a large fraction of $\mathrm{HI}$ is unrelated to the cold phase traced by $\mathrm{H}_{2}$. Finally, our understanding of the formation of $\mathrm{H}_{2}$ onto dust grains, self-shielding, and lifetime of cold diffuse gas would certainly benefit from further observations of chlorine and molecular hydrogen in different environments and over a wide range of column densities.

Acknowledgements. S.B. and V.K. thank RF President Program (grant MK4861.2013.2) and "Leading Scientific Schools of Russian Federation" (grant NSh-294.2014.2). R.S. and P.P. gratefully acknowledge support from the IndoFrench Centre for the Promotion of Advanced Research (Centre Franco-Indien pour la Promotion de la Recherche Avancée) under contract No. 4304-2.

\section{References}

Albornoz Vásquez, D., Rahmani, H., Noterdaeme, P., et al. 2014, A\&A, 562, A88

Asplund, M., Grevesse, N., Sauval, A. J., \& Scott, P. 2009, ARA\&A, 47, 481

Balashev, S. A., Ivanchik, A. V., \& Varshalovich, D. A. 2010, Astron. Lett., 36, 761

Balashev, S. A., Petitjean, P., Ivanchik, A. V., et al. 2011, MNRAS, 418, 357

Balashev, S. A., Klimenko, V. V., Ivanchik, A. V., et al. 2014, MNRAS, 440, 225

Carswell, R. F., Jorgenson, R. A., Wolfe, A. M., \& Murphy, M. T. 2011, MNRAS, 411, 2319

Dunlop, J. S. 2011, Science, 333, 178

Guimarães, R., Noterdaeme, P., Petitjean, P., et al. 2012, AJ, 143, 147

Harris, A. W., \& Bromage, G. E. 1984, MNRAS, 208, 941

Jenkins, E. B. 2009, ApJ, 700, 1299

Jenkins, E. B., Savage, B. D., \& Spitzer, Jr., L. 1986, ApJ, 301, 355

Jorgenson, R. A., Wolfe, A. M., Prochaska, J. X., \& Carswell, R. F. 2009, ApJ, 704,247

Jorgenson, R. A., Wolfe, A. M., \& Prochaska, J. X. 2010, ApJ, 722, 460

Jura, M. 1974, ApJ, 190, L33

Kanekar, N., Prochaska, J. X., Smette, A., et al. 2014, MNRAS, 438, 2131

Krogager, J.-K., Fynbo, J. P. U., Møller, P., et al. 2012, MNRAS, 424, L1

Ledoux, C., Petitjean, P., Bergeron, J., Wampler, E. J., \& Srianand, R. 1998, A\&A, 337, 51

Ledoux, C., Srianand, R., \& Petitjean, P. 2002, A\&A, 392, 781

Ledoux, C., Petitjean, P., \& Srianand, R. 2003, MNRAS, 346, 209

Ledoux, C., Petitjean, P., \& Srianand, R. 2006, ApJ, 640, L25

Levshakov, S. A., \& Varshalovich, D. A. 1985, MNRAS, 212, 517

Lis, D. C., Pearson, J. C., Neufeld, D. A., et al. 2010, A\&A, 521, L9

Malec, A. L., Buning, R., Murphy, M. T., et al. 2010, MNRAS, 403, 1541

Moomey, D., Federman, S. R., \& Sheffer, Y. 2012, ApJ, 744, 174

Morton, D. C. 2003, ApJS, 149, 205

Neufeld, D. A., \& Wolfire, M. G. 2009, ApJ, 706, 1594

Noterdaeme, P., Ledoux, C., Petitjean, P., et al. 2007a, A\&A, 474, 393

Noterdaeme, P., Petitjean, P., Srianand, R., Ledoux, C., \& Le Petit, F. 2007b, A\&A, 469, 425

Noterdaeme, P., Ledoux, C., Petitjean, P., \& Srianand, R. 2008, A\&A, 481, 327

Noterdaeme, P., Petitjean, P., Ledoux, C., \& Srianand, R. 2009, A\&A, 505, 1087

Noterdaeme, P., Petitjean, P., Ledoux, C., et al. 2010, A\&A, 523, A80

Noterdaeme, P., Petitjean, P., Srianand, R., Ledoux, C., \& López, S. 2011, A\&A, 526, L7

Noterdaeme, P., Srianand, R., Rahmani, H., et al. 2015, A\&A, accepted [arXiv: 1502 .03921]

Oliveira, C. M., \& Hébrard, G. 2006, ApJ, 653, 345

Petitjean, P., Srianand, R., \& Ledoux, C. 2000, A\&A, 364, L26

Petitjean, P., Ledoux, C., Noterdaeme, P., \& Srianand, R. 2006, A\&A, 456, L9

Prochaska, J. X., \& Wolfe, A. M. 2009, ApJ, 696, 1543

Prochaska, J. X., Howk, J. C., \& Wolfe, A. M. 2003, Nature, 423, 57

Rafelski, M., Wolfe, A. M., Prochaska, J. X., Neeleman, M., \& Mendez, A. J. 2012, ApJ, 755, 89

Savage, B. D., \& Sembach, K. R. 1996, ARA\&A, 34, 279

Schectman, R. M., Federman, S. R., Beideck, D. J., \& Ellis, D. J. 1993, ApJ, 406, 735

Sonnentrucker, P., Friedman, S. D., \& York, D. G. 2006, ApJ, 650, L115

Srianand, R., Petitjean, P., Ledoux, C., Ferland, G., \& Shaw, G. 2005, MNRAS, 362,549

Srianand, R., Noterdaeme, P., Ledoux, C., \& Petitjean, P. 2008, A\&A, 482, L39

Srianand, R., Gupta, N., Petitjean, P., et al. 2012, MNRAS, 421, 651

Srianand, R., Gupta, N., Rahmani, H., et al. 2013, MNRAS, 428, 2198 